http://jmscr.igmpublication.org/home/

ISSN (e)-2347-176x ISSN (p) 2455-0450

crossref DOI: https://dx.doi.org/10.18535/jmscr/v8i7.66

Journal Of Medical Science And Clinical Research

IGM Publication

An Official Publication of IGM Publication

\title{
Fatal Pancreatic Transection in Pediatric Blunt Abdominal Trauma
}

Authors

\section{Dr Gerard Pradeep Devnath, M.B.B.S., M.D ${ }^{1}$, Dr Kirthika Ravi, M.B.B.S., M.D ${ }^{2 *}$, Dr Ajoy Kumar Ghosh, M.B.B.S., M.D ${ }^{3}$}

${ }^{1}$ Senior Resident, Department of Forensic Medicine and Toxicology, Jawaharlal Institute of Postgraduate Medical Education \& Research, Puducherry, India, 605006

${ }^{2,3}$ Junior Resident, Department of Forensic Medicine and Toxicology, Jawaharlal Institute of Postgraduate Medical Education \& Research, Puducherry, India, 605006

*Corresponding Author

Dr Kirthika Ravi, M.B.B.S., M.D

Junior Resident, Department of Forensic Medicine and Toxicology, Jawaharlal Institute of Postgraduate Medical Education \& Research, Puducherry, India, 605006

\begin{abstract}
Road traffic accidents are the leading cause of death among children and young people aged between 529 years. Pancreatic injury following a blunt trauma abdomen is an uncommon presentation. Pancreatic injury is generally associated with other intra-abdominal organ injuries and are often difficult to diagnose initially at the emergency setup. We report a fatal case of grade IV pancreatic injury that is pancreatic transection at its body following a blunt trauma abdomen. Mortality in paediatric pancreatic injury is less compared to the morbidity. This case report features gross and computed tomography image which may give a much better understanding of paediatric pancreatic injuries.

Keywords: Paediatric trauma; road traffic accidents; pancreas; blunt abdominal trauma.
\end{abstract}

\section{Background}

Globally 1.35 million people die due to road traffic accidents every year. Road traffic accidents leading cause of death among children and young people aged between 5-29 years. The commonest cause of death in children in developed countries is trauma, accounting for over 350 deaths/year in the UK. In children, about $75 \%$ of trauma occurs on the roads and the majority of these involve blunt trauma including falls, road traffic accidents (RTA), and bicycle-related injuries. Road traffic accidents accounts for $42 \%$ blunt trauma to abdomen in children. ${ }^{1}$ Incidence of pancreatic injury is $0.3-0.4$ per cent in the paediatric population. Mortality in the paediatric pancreatic injury varies from 3 to $17 \% .^{2}$ Traumatic pancreatic injury commonly involves at the junction of body and tail and less commonly head. Pancreatic injury following a blunt trauma abdomen is often an uncommon presentation and difficult to diagnose initially. This case highlights complete pancreatic injury in blunt trauma to the abdomen. Generally, pancreatic injury is seen in penetrative injuries. This case demonstrates pancreatic transection following blunt trauma abdomen due to a road traffic accident. This case 
features gross and radiological findings and mechanism of pancreatic injury and so that it may provide much better information for the treating physician.

\section{Case Report}

A five-year-old-female child, presented with a history of road traffic accident while travelling in a car. The child was seated in the rear seat row along with 3 occupants. The child was not restrained with a seat belt. The car along with the occupants toppled and rolled over few times before falling into a ditch and the child was thrown out through the window. The child presented to the emergency department with complaints of vomiting and abdominal pain. The child was conscious, oriented with a GCS-15/15. On systemic examination, diffuse tenderness noted per abdomen. Serum amylase 746 and lipase 2045 IU/L during admission. After 6 hours the Serum amylase and lipase 4456 and 7027 IU/L respectively. Peritoneal fluid was haemorrhagic and WBC count was 3200, neutrophils 70\%, lymphocytes $20 \%$ and monocytes $10 \%$. CT abdomen showed complete transection of the pancreas with hemoperitoneum associated with liver injury. The child expired within 18 hours of treatment. In post-mortem examination externally, contusion of size $(7 \mathrm{cmX} 2 \mathrm{~cm})$ was present over the anterior abdominal wall placed $7 \mathrm{~cm}$ above the umbilicus. A contusion of size $(3 \mathrm{cmX} 2 \mathrm{~cm})$ present over left iliac fossa. Multiple abrasions present over the back of the trunk. Around $300 \mathrm{ml}$ blood was present in the peritoneal cavity. Retroperitoneal hematoma weighing 400cc was present covering the pancreas. On removing the clot, pancreas showed complete transection at the middle one-third of the body placed $2 \mathrm{~cm}$ from the head. Liver noted with a sub-capsular contusion of $(6.5 \mathrm{~cm} \mathrm{X} 1 \mathrm{~cm})$ over the posterior aspect of the left lobe. Around $250 \mathrm{ml}$ of blood-stained fluid present in the bilateral pleural cavity. All organs were found to be intact and on cut section congested.

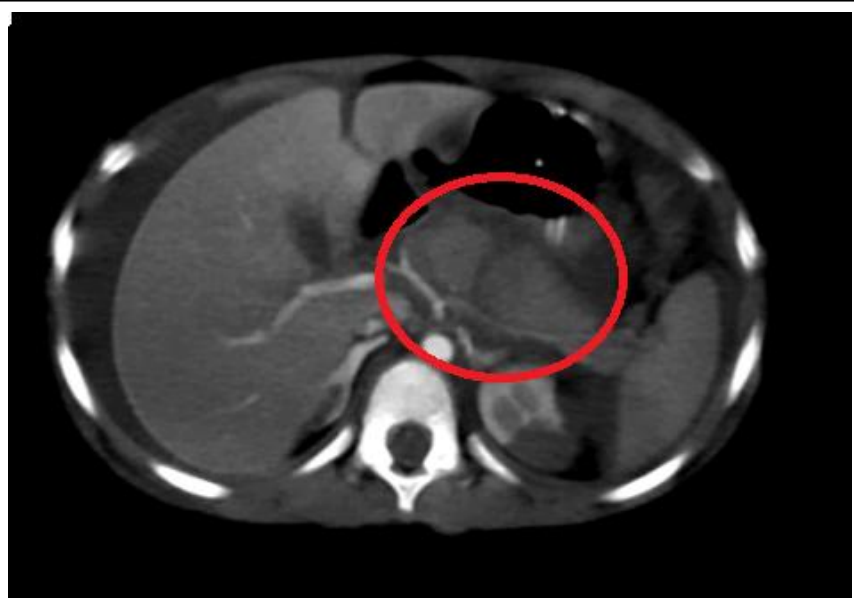

Fig 1: CT abdomen showing pancreatic transection

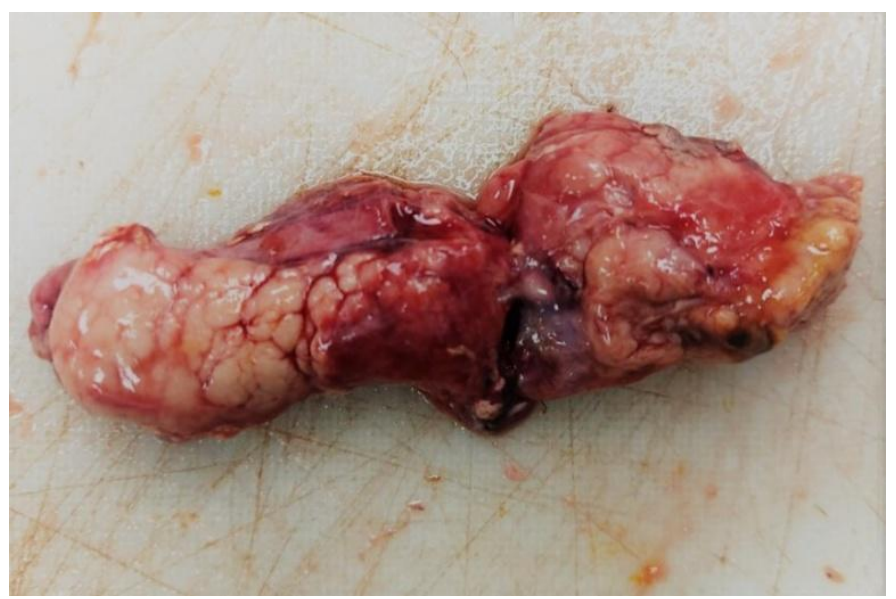

Fig 2: proximal transection at the body of pancreas

\section{Discussion}

Paediatric population are vulnerable to pancreatic injuries due to its anatomical positioning. The flat diaphragm and malleable ribs position the liver and spleen more anteriorly thus possibly crushing solid organs like pancreas below. Pancreas is prone to injury due to less abdominal fat and peripancreatic fat covering in children, unlike adults. ${ }^{3,4}$ A sudden and localized force transmitted to the upper abdomen compresses the pancreas against the vertebral body which leads to contusion and laceration in different sites depending on the direction and force of injury sustained. Paediatric pancreatic injury may be due to blunt trauma abdomen or penetrative injuries. Blunt trauma includes bicycle-related injuries (handlebar injury) road traffic accidents and falls. Penetrative injuries include stab injury, gunshot 
and impalement injuries. Pancreas is the fourth most common organ to be injured (2 to 9\%) in blunt trauma following the kidneys, spleen and liver. ${ }^{5}$ Englum BR et al, assessed the data of 610,402 paediatric trauma cases from the National Trauma Data Bank (NTDB) from 2007-2011. Around 1653 cases $(0.3 \%)$ had blunt pancreatic injury of which 514 cases $(76.3 \%)$ had nonoperative pancreatic management. The morbidity and mortality rates were $26.5 \%$ and $5.3 \%$ respectively. ${ }^{6}$ Morbidity is high in paediatric pancreatic injury due to complications sustained like pseudocysts, ascites, ductal stricture and pancreatic insufficiency. ${ }^{7}$ Pancreatic injuries present immediately or late with vague, subtle features which is the reason behind late diagnosis and management. Generally, pancreatic injuries are associated with other organ injuries like spleen, liver and kidneys, which was noted in this case too. ${ }^{8}$

As per the American Association for the Surgery of Trauma (AAST) grading for pancreatic injury, this case falls under grade IV (Distal transection or pancreatic parenchymal injury with ductal injury). ${ }^{9}$ The CT based grading of this injury fall under Grade C-II, i.e., proximal pancreas transection

This case highlights the pancreatic transection in a case of blunt trauma abdomen following a road traffic accident in a child. Fatality in paediatric pancreatic injuries are very less. The incidence of Grade IV pancreatic injury is uncommon in the medical literature.

\section{Conclusion}

Pancreatic transection in a blunt trauma is an uncommon manifestation in children. Data on Grade IV paediatric pancreatic injury is minimal. Mortality in paediatric pancreatic injuries based on the grade of injury is spare and hence this autopsy based case report can contribute significant data to the medical literature.
Conflict of Interest: The authors don't have any conflict of interest to disclose in relation to this paper.

\section{Funding: None}

\section{References}

1. Road Traffic Injuries and Deaths-A Global Problem. National Center for Injury Prevention and Control (NCIPC). Centers for Disease Control and Prevention (CDC). Accessed on 20/03/2020. Available from URL: https://www.cdc.gov/injury/features/global -road-safety/index.html

2. Debi $U$ et al. Pancreatic trauma: a concise review. World J Gastroenterol. 2013; 19(47): 9003-90.

3. Gaines BA and Ford HR. Abdominal and pelvic trauma in children. Critical care medicine. 2002;30(11):416-23.

4. Gupta A et al. Blunt trauma of the pancreas and biliary tract: a multimodality imaging approach to diagnosis. Radiographics. 2004;24(5):1381-95.

5. Jobst MA et al. Management of pancreatic injury in pediatric blunt abdominal trauma. J Pediatr Surg Case Rep. 1999;34(5):81824.

6. Englum BR et al. Management of blunt paediatric trauma in Children: Review of the National trauma Data Bank. J Pediatric Surg. 2016; 51(9): 1526-1531.

7. Al-Ahmadi K and Ahmed N. Outcomes after pancreatic trauma: experience at a single institution. Can J Surg 2008;51(2): $118 \mathrm{e} 24$.

8. Taylor CW and Loveland J. Paediatric pancreatic trauma: A review of literature and results of multicenter survey on patient management. S Atr Med J. 2014; 104(11): 803-807.

9. Wong YC et al. CT grading of blunt pancreatic injuries: prediction of ductal disruption and surgical correlation. $\mathbf{J}$ Comput Assist Tomogr. 1997;21:246-250. 